\title{
Faune mammalienne, chasse et conflits humains- faune en périphérie du Parc national de Kundelungu (R.D. Congo)
}

\author{
Kazaba Kaseya Paul1,4*, Bahongoli Mondjono Maurice, Kilemba Mukangala Benjamin², Ankwanda \\ Yungu Albert ${ }^{1}$, Tshikung Kambol Mosses Didier ${ }^{3}$ \\ 1 Unité de Recherche en Ecologie, Restauration écologique et Paysage (EREP), Faculté des Sciences agronomiques, \\ Université de Lubumbashi, R.D. Congo. \\ 2Unité de Recherche en Nutrition, Amélioration animale et Agropastoralisme, Faculté des Sciences agronomiques, \\ Université de Lubumbashi, R.D. Congo. \\ 3Département des Sciences de Base, Faculté de Médecine vétérinaire, Université de Lubumbashi, R.D. Congo. \\ ${ }^{4}$ Department of Environmental Management, Pan African University Life and Earth Sciences Institute (PAULESI), \\ University of Ibadan, Nigeria. \\ *Auteur correspondant : paulkazaba@gmail.com
}

Original submitted in on $14^{\text {th }}$ February 2019. Published online at www.m.elewa.org/journals/ on $31^{\text {st }}$ July 2019 https://dx.doi.org/10.4314/jab.v139i1.2

\section{RESUME}

Objectifs : Cette étude a cherché à répertorier la faune mammalienne et à rassembler les informations préliminaires sur la chasse et les conflits humains-faune en périphérie du Parc national de Kundelungu.

Méthodologie et résultats : La recherche s'est appuyée sur des observations directes et des enquêtes avec questionnaires conduites auprès d'un échantillon de 120 répondants dans quatre localités sélectionnées sur base de leur proximité à la fois au Parc et à des écosystèmes relativement intacts. II en ressort que parmi les animaux vus en milieu naturel entre une semaine et trois mois avant les enquêtes, les répondants ont listé un total de 24 espèces, principalement des rongeurs et des artiodactyles. La plupart de ces espèces font l'objet d'une chasse de subsistance et sont réputés nuisibles aux cultures.

Conclusions et application: Plusieurs espèces répertoriées indiquent que la zone périphérique du Parc présente les caractéristiques des milieux perturbés, où l'espace géographique est partagé entre la faune et les populations locales. La principale leçon tirée de cette étude et, éventuellement, à appliquer à bien d'autres aires protégées en Afrique est que le succès de la conservation nécessitera non seulement l'implication de ces populations et des actions concertées dans la gestion des conflits humains-faune, mais également la caractérisation des activités agricoles et des stratégies locales de lutte contre les animaux réputés ravageurs des cultures dans les environs des aires protégées. Ceci permettra d'identifier les chasseurs occasionnels et potentiels et ainsi de mieux évaluer les menaces d'origine anthropiques sur des zones d'intérêt écologique mais ne jouissant pas de mesures strictes de protection.

Mots clés : faune mammalienne, chasse, conflits humains-faune, conservation, aires protégées, HautKatanga 


\section{ABSTRACT \\ Mammals, Hunting and Human-Wildlife Conflicts in the Periphery of Kundelungu National Park, D.R. Congo}

Objectives: This study aimed at identifying mammals, characterizing hunting activities and analyzing human-wildlife conflicts on the periphery of Kundelungu National Park.

Methodology and Results: Data were collected through direct observations and questionnaire surveys conducted with a sample of 120 respondents in four villages chosen on the basis of their proximity to both the Park and relatively intact ecosystems. Among the animals seen in the wild between one week and three months before the survey, respondents listed 24 species, mainly rodents and artiodactyls. Most of these species are hunted and are deemed to cause damages to crops.

Conclusions and Application: A number of species listed by respondents indicate that the periphery of the Park has some characteristics of disturbed environments shared between wildlife and local people. The main lesson from this study (and possibly applicable to many other protected areas in Africa) is that achieving conservation goals will require not only the involvement of local people and collaborative actions in addressing human-wildlife conflicts, but also the characterization of agricultural activities and local strategies used to repel pest animals in the vicinities of protected areas. This will identify both occasional and potential hunters and hence accurately quantify human-driven threats on ecologically important zones that are not protected as strictly as required.

Keywords: mammals, hunting, human-wildlife conflicts, conservation, protected areas, Upper Katanga

\section{INTRODUCTION}

Plusieurs études ont montré qu'à travers le monde, les aires protégées ne parviendront pas à elles seules à endiguer la perte de biodiversité, notamment à cause de leur faible couverture spatiale et des conflits avec les activités humaines dans leurs périphéries (Inogwabini et al., 2005; Mora \& Sale 2011; Geldmann et al., 2013; Moussa et Amadou, 2014). Le rôle des périphéries des aires protégées dans la conservation de la biodiversité a été discuté par plusieurs auteurs. En Afrique, les conclusions de la plupart des travaux convergent sur deux idées majeures: (i) des espaces naturels aux abords des aires protégées sont d'une importance écologique indéniable, notamment par l'importante diversité biologique qu'elles regorgent et par leurs contributions aux processus écologiques (Dupain et al., 2004; Western et al.,2009; Soulama et al., 2015; Jiagho et al., 2016); (ii) les activités humaines en périphérie peuvent constituer une menace pour les ressources naturelles conservées au sein même des aires protégées (Balme et al., 2010; Baudron et al., 2011; Avakoudjo et al.,2014; Soulama et al., 2015).
Avec sa flore, sa faune diversifiée et ses habitats naturels, dont plus de 150 millions d'hectares de forêts, représentant près de $60 \%$ de la superficie forestière du Bassin du Congo, la République démocratique du Congo (RDC) a un rôle important à jouer dans le maintien des équilibres écologiques et de la biodiversité sur la planète. Depuis 1925 déjà, le pays a amorcé la mise en place d'un vaste réseau d'aires protégées (Inogwabini et al., 2005) pour, principalement, préserver cette faune et ces habitats. Cependant, au moment où la RDC s'emploie à étendre son réseau d'aires protégées terrestres de sorte qu'il atteigne au moins $17 \%$ de la superficie de son territoire à l'horizon 2020 (Objectif 11 d'Aichi), des défis de tous ordres restent à relever pour assurer l'efficacité des mesures de conservation au sein même des parcs et réserves existants (Inogwabini et al., 2005; UICN, 2010). Une situation encore plus alarmante s'observe dans les périphéries de ces aires où des mesures appropriées ne sont pas encore arrêtées en réponse à l'essor des activités humaines menaçant la faune et les habitats naturels. 
La présente étude a concerné les alentours du Parc national de Kundelungu au sud-est de la RDC, avec comme objectifs spécifiques de: (i) répertorier les mammifères, animaux parmi les plus ciblés par la chasse et le commerce de viande de brousse; (ii) caractériser les activités de chasse, et (iii) analyser les conflits humains-faune. Son but était de rassembler des données préliminaires pouvant

\section{MATERIEL ET METHODES}

Zone d'étude : L'étude a été conduite aux environs de la partie sud du Parc national de Kundelungu, dans la province du Haut-Katanga au sud-est de la RDC (Figure 1). A l'issue des pré-enquêtes conduites entre janvier et février 2017 dans cette zone, quatre villages ont été sélectionnés sur base de leur proximité au Parc et de la présence des animaux sauvages et des forêts orienter la mise au point d'outils de gestion durable de ce qui reste de la faune dans cette zone comprenant des forêts qui, en vertu du Code forestier de la RDC (Anonyme, 2002), «n'ont pas fait l'objet d'un acte de classement et sont soumises à un régime juridique moins restrictif quant aux droits d'usage et aux droits d'exploitation».

relativement intactes dans leurs environs. II s'agit de:

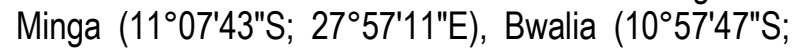
$\left.28^{\circ} 10^{\prime} 08^{\prime \prime} \mathrm{E}\right)$, Malambwe $\left(11^{\circ} 15^{\prime} 52^{\prime \prime S}\right.$; $\left.27^{\circ} 52^{\prime} 25^{\prime \prime E}\right)$ et Kasomeno $\left(10^{\circ} 45^{\prime} 20^{\prime \prime S} ; \quad 28^{\circ} 17^{\prime} 04^{\prime \prime} \mathrm{E}\right)$, situés respectivement à $5,7 \mathrm{~km}, 6 \mathrm{~km}, 23 \mathrm{~km}$ et $24 \mathrm{~km}$ de la limite la plus proche du Parc.

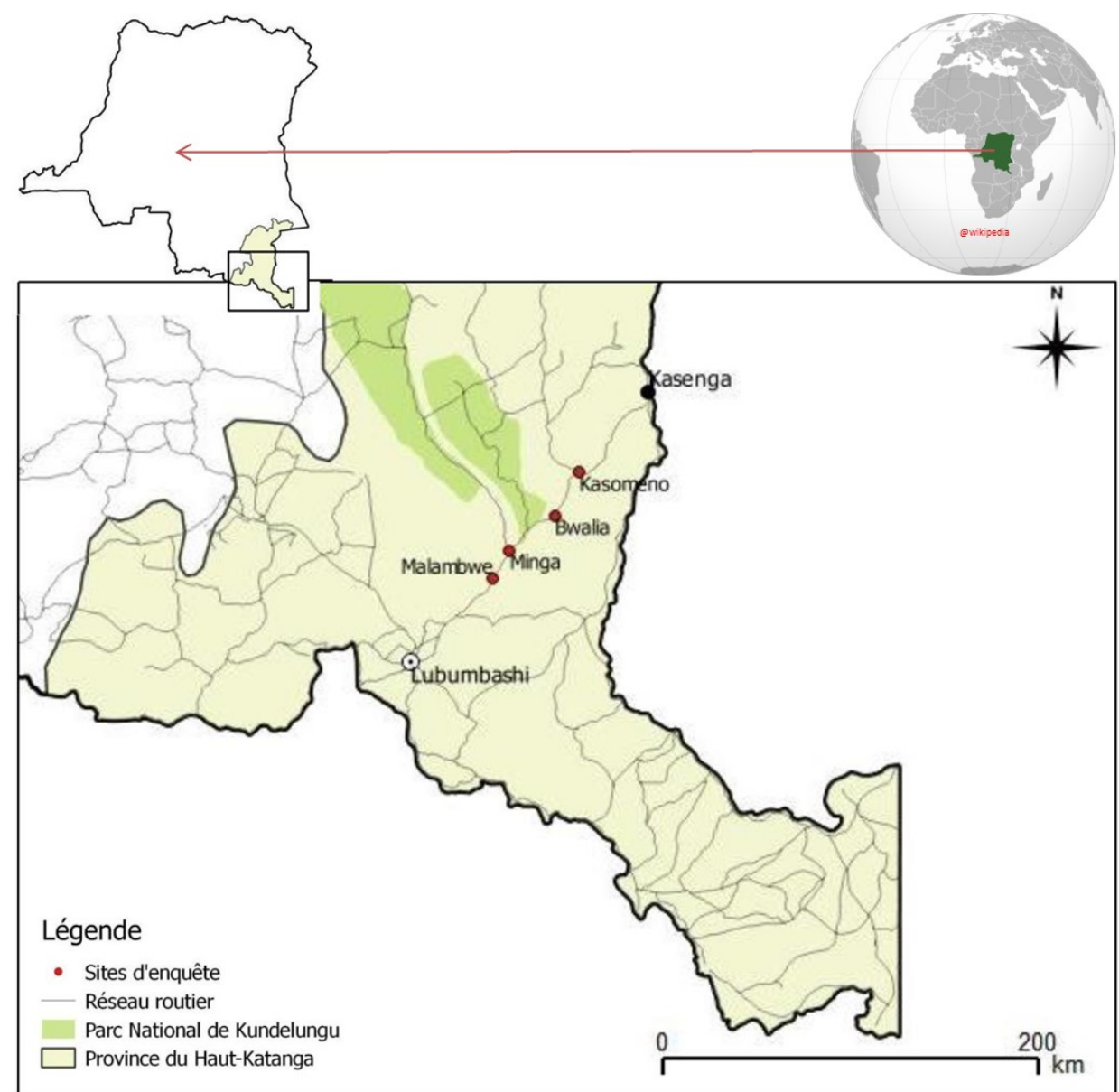

Figure 1: Localisation du Parc national de Kundelungu et des quatre villages retenus pour les enquêtes au sud-est de la République Démocratique du Congo. 
La zone d'étude est sous un climat tropical caractérisé par une saison pluvieuse allant de novembre à mars et une saison sèche de mai à septembre, octobre et avril étant considérés comme des mois de transition (Assani, 1999). D'une moyenne annuelle de $20^{\circ} \mathrm{C}$, les températures y varient de 16 à $33^{\circ} \mathrm{C}$; la moyenne annuelle des précipitations est d'environ $1300 \mathrm{~mm}$ (Saad et al., 2012). L'ensemble de cette zone fait partie du centre d'endémisme zambézien (White, 1983) et d'un paysage anthropisé aux abords de Lubumbashi où des lambeaux de forêts claires du type Miombo (Malaisse, 2010) subsistent entre savanes, terres cultivées et villages (Kabulu et al., 2008; Munyemba \& Bogaert, 2014).

Collecte et analyse des données : Les données ont été rassemblées au cours du mois d'août 2017 grâce à une enquête avec questionnaire complétée par des observations directes sur terrain. Des questions ouvertes et fermées ont été adressées à un échantillon de 120 répondants appartenant à des ménages différents et choisis au hasard parmi les personnes âgées de plus de 30 ans fréquentant les périphéries du Parc national de Kundelungu et donc ayant une connaissance suffisante de sa faune et une perception judicieuse de la dynamique des populations animales. En raison de l'absence des données concordantes et précises sur le nombre d'habitants, la taille de l'échantillon a été fixée à 120 , soit une moyenne de 30 enquêtés par village, au lieu d'être calculée au moyen des formules statistiques usuelles.

Les répondants à l'enquête ont listé les animaux sauvages couramment observés avant de fournir des informations sur les activités de chasse et les interactions humains-faune dans la zone. Une attention

\section{RESULTATS}

Mammifères observés récemment dans la périphérie sud du Parc national de Kundelungu : En ce qui concerne les mammifères observés en milieu naturel peu avant l'enquête, 24 espèces appartenant à cinq ordres (Tableau 1 et Figure 2) ont été répertoriés de manière cumulative par les 120 répondants. Avec le rat géant de Gambie (Cricetomys gambianus), le rat du Veld commun (Otomys typus) et l'écureuil de Smith (Paraxerus cepapi) figurant parmi les espèces les plus citées, les rongeurs sont l'ordre le plus représenté particulière a été accordée au dernier mammifère observé en milieu naturel dans les environs du Parc. Hormis les noms vernaculaires et les descriptions morphologiques de cet animal, les répondants ont donné des indications sur le lieu d'observation et le temps écoulé depuis qu'il a été vu dans la nature, la date des enquêtes étant prise comme référence. L'identification des animaux mentionnés au niveau de l'espèce a été faite au moyen d'un guide illustré répertoriant les mammifères africains avec leurs aires de répartition (Kingdon, 2008) et sur base de trois types d'informations: (i) les noms locaux des animaux (en Swahili, Bemba ou Lamba, selon les cas) et les descriptions morphologiques fournies par les répondants; (ii) quelques trophées de chasse (dont notamment des peaux, cranes et cornes d'antilopes) conservés par certains répondants, et (iii) les indices de présence de ces animaux sur terrain. Dans certains cas où ces informations n'étaient pas suffisantes pour déterminer les espèces avec précision, l'identification a été limitée à un rang taxonomique supérieur tel que le genre.

Les données collectées ont fait l'objet des statistiques descriptives dans MS Office Excel. Pour des questions fermées telles que celle relative au temps écoulé depuis la dernière observation d'un mammifère, il a été question de comparer les distributions des réponses sur des diagrammes à barres. D'autres distributions ont également été comparées afin de ressortir les espèces et groupes taxonomiques les plus représentés. La significativité des différences entre les proportions des réponses renvoyant aux espèces et groupes d'espèces a été vérifiée au moyen d'un test du Chi-carré, au seuil de $5 \%$.

(mentionnés par $40 \%$ des 120 répondants; $X^{2}=28, P<$ $0,001)$. Ce groupe est suivi des artiodactyles, ordre au sein duquel le guib harnaché (Tragelaphus scriptus), le céphalophe bleu (Philantomba monticola) et le céphalophe de Grimm (Sylvicapra grimmia) ont été les plus mentionnés. Seulement $7 \%$ des répondants ont fait référence aux primates, représentés par le Galago à queue touffue (Otolemur crassicaudatus) et le babouin de Kinda (Papio kindae). 


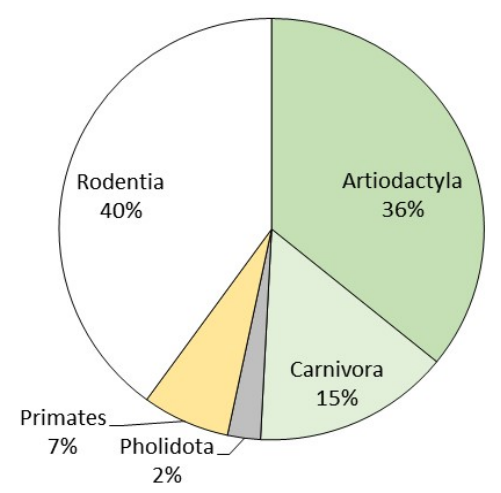

Figure 2: Groupes d'animaux et proportions des 120 répondants les ayant mentionnés dans les environs du Parc national de Kundelungu.

Le temps écoulé entre le moment de l'enquête (17-21 août 2017) et le jour où le dernier animal a été observé dans la nature donne une indication sur l'abondance de la faune. Les estimations de ce temps par les enquêtés observe dans l'ensemble que pour la majorité (60\%) des 120 répondants, un mammifère a été vu en milieu naturel au cours d'une période de moins d'un mois ont connu la distribution présentée sur la Figure 3. II s'y avant les enquêtes.

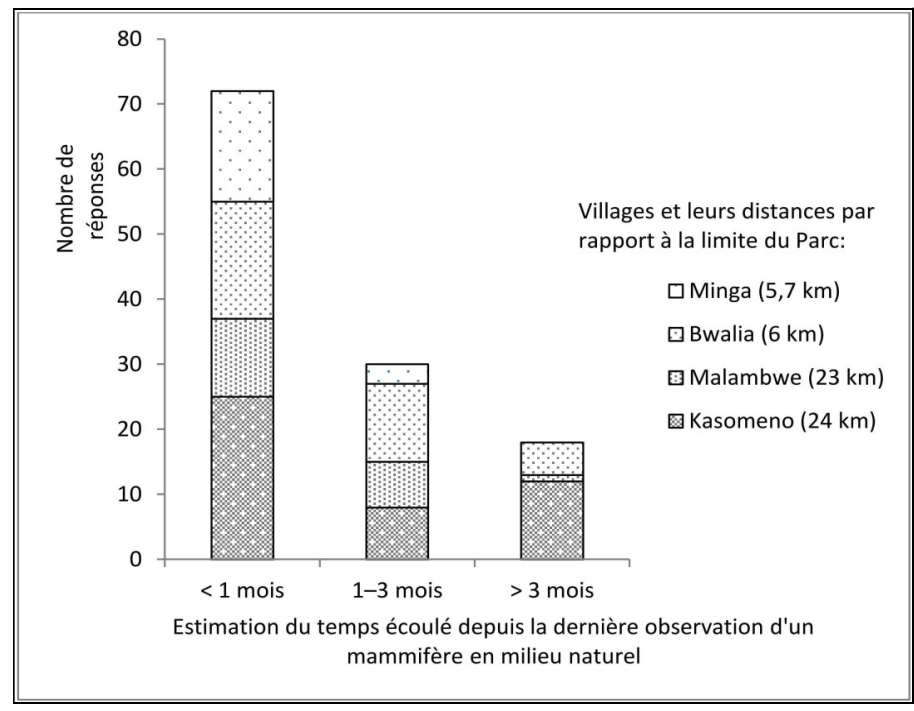

Figure 3: Distribution des estimations par les 120 répondants du temps écoulé depuis l'observation d'un mammifère dans les environs des quatre villages, la période d'enquête (17-21 août 2017) étant prise comme référence. Les distances entre parenthèses sont mesurées entre le centre du village et la limite la plus proche du Parc national de Kundelungu.

En outre, en ce qui conserve de l'état de conservation (Tableau 1), sur l'ensemble des 24 espèces répertoriées, 21 sont dans la catégorie «Préoccupation mineure» (LC) sur la liste rouge de l'Union Internationale pour la Conservation de la Nature (UICN).
Seule la loutre à joues blanches (Aonyx capensis) est listée "Quasi-menacée»; le pangolin à petites écailles (Phataginus tricuspis) et l'hippopotame amphibie (Hippopotamus amphibius) sont quant à eux évalués «Vulnérable» (VU). 
Tableau 1: Liste des mammifères déclarés vus par 120 répondants dans les environs de quatre localités en périphérie sud du Parc national de Kundelungu.

\begin{tabular}{|c|c|c|c|c|c|c|}
\hline \multicolumn{2}{|l|}{ Espèce } & \multirow{2}{*}{ Famille } & \multirow{2}{*}{ Ordre } & \multirow{2}{*}{$\begin{array}{l}\text { Proportion des } \\
\text { répondants (\%) }\end{array}$} & \multirow{2}{*}{$\begin{array}{l}\text { Statut global } \\
\text { (UICN, 2018) }\end{array}$} & \multirow{2}{*}{$\begin{array}{l}\text { Statut juridique en RDC } \\
\text { (Anonyme, 2004) }\end{array}$} \\
\hline Nom scientifique & Nom commun & & & & & \\
\hline Cricetomys gambianus & Rat géant de Gambie & Nesomyidae & Rodentia & 14,17 & LC & Non encore protégé \\
\hline Tragelaphus scriptus & Guib harnaché & Bovidae & Artiodactyla & 12,50 & LC & $\begin{array}{l}\text { Partiellement protégé } \\
\text { (Tableau 2) }\end{array}$ \\
\hline Sylvicapra grimmia & Céphalophe de Grimm & Bovidae & Artiodactyla & 11,67 & LC & Non encore protégé \\
\hline Philantomba monticola & Céphalophe bleu & Bovidae & Artiodactyla & 9,17 & LC & Non encore protégé \\
\hline Otomys typus & Rat du Veld commun & Muridae & Rodentia & 7,50 & LC & Non encore protégé \\
\hline Canis adustus & Chacal à flancs rayés & Canidae & Carnivora & 5,83 & LC & Non encore protégé \\
\hline Otolemur crassicaudatus & Galago à queue touffue & Galagidae & Primates & 5,00 & LC & Tableau 2 \\
\hline Paraxerus cepapi & Ecureuil de Smith & Sciuridae & Rodentia & 5,00 & LC & Non encore protégé \\
\hline Thryonomys swinderianus & Grand aulacode & Thryonomyidae & Rodentia & 4,17 & LC & Non encore protégé \\
\hline Hystrix africaeaustralis & $\begin{array}{l}\text { Porc-épic d'Afrique } \\
\text { australe }\end{array}$ & Hystricidae & Rodentia & 3,33 & LC & Non encore protégé \\
\hline Atilax paludinosus & Mangouste des marais & Herpestidae & Carnivora & 2,50 & LC & Non encore protégé \\
\hline Phataginus tricuspis & $\begin{array}{l}\text { Pangolin à petites } \\
\text { écailles }\end{array}$ & Manidae & Pholidota & 2,50 & VU & Tableau 2 \\
\hline Cryptomys hottentotus & Rat-taupe africain & Bathyergidae & Rodentia & 2,50 & LC & - \\
\hline Papio kindae & Babouin de Kinda & Cercopithecidae & Primates & 1,67 & LC & Non encore protégé \\
\hline Civettictis civetta & Civette africaine & Viverridae & Carnivora & 1,67 & LC & Non encore protégé \\
\hline Felis serval & Serval & Felidae & Carnivora & 1,67 & LC & Tableau 2 \\
\hline Mastomys natalensis & $\begin{array}{l}\text { Rat du Natal (rat africain } \\
\text { commun) }\end{array}$ & Muridae & Rodentia & 1,67 & LC & - \\
\hline Poecilogale albinucha & Zorille à nuque blanche & Mustelidae & Carnivora & 1,67 & LC & - \\
\hline Potamochoerus larvatus & Potamochère & Suidae & Artiodactyla & 1,67 & LC & - \\
\hline Anomalurus derbianus & Anomalure de Derby & Anomaluridae & Rodentia & 0,83 & LC & - \\
\hline Aonyx capensis & Loutre à joues blanches & Mustelidae & Carnivora & 0,83 & NT & - \\
\hline Felis sylvestris & Chat sauvage & Felidae & Carnivora & 0,83 & LC & - \\
\hline Helioscirus gambianus & $\begin{array}{l}\text { Grand écureuil de } \\
\text { Gambie }\end{array}$ & Sciuridae & Rodentia & 0,83 & LC & - \\
\hline Hippopotamus amphibius & Hippopotame amphibie & Hyppopotamidae & Artiodactyla & 0,83 & VU & Tableau 2 \\
\hline
\end{tabular}

Catégories UICN des espèces: LC = Least Concern (Préoccupation mineure); NT = Near Threatened (Quasi-menacé); VU = Vulnérable. 
Activités de chasse : Des données sur les activités humaines dans la zone, il sied de noter que l'agriculture a été mentionnée par environ $88 \%$ des répondants comme activité principale, alors que la chasse n'a été citée que par seulement $5 \%$ d'entre eux, et ce comme activité secondaire. Cette chasse, pratiquée en dehors des aires et des périodes fixées par la loi, est motivée essentiellement par la satisfaction des besoins alimentaires. En effet, la quasi-totalité des répondants ont mentionné l'approvisionnement en viande de brousse comme principale fonction de la faune du milieu. Mentionnés respectivement par environ 26 et $10 \%$ des répondants, l'aulacode (Thryonomys swinderianus) et le rat géant de Gambie sont les principaux gibiers de la zone au cours de ces dernières années. En dehors des autres mammifères fréquemment observés dans le milieu (Tableau 1), parmi les gibiers listés, il y a aussi des reptiles tels que le varan du Nil (Varanus niloticus) et des tortures terrestres, et des oiseaux, notamment des passeriformes chassés au filet.

La plupart des animaux faisant l'objet de la chasse dans la zone ne bénéficient pas des mesures strictes de protection. En effet, au regard des mesures d'exécution de la loi portant réglementation de la chasse en RDC (Anonyme, 2004), sur les 24 espèces répertoriées lors des enquêtes, aucune n'est totalement protégée (ne figurant pas au Tableau 1), et seulement cinq sont visées au Tableau 2 (espèces partiellement protégées). Le reste ne sont reprises sur aucun des Tableaux et peuvent faire l'objet de la chasse dans des conditions beaucoup moins strictes.

Cohabitation et conflits humains-faune : Les données collectées ont permis d'analyser la question

\section{DISCUSSION}

L'importante proportion des répondants ayant mentionné des espèces de rongeurs et d'artiodactyles pourrait indiquer une prépondérance de ces espèces par rapport aux autres mammifères en périphérie du Parc national de Kundelungu. Dans plusieurs parties de la RDC ou dans le Bassin du Congo en général, les informations que ce soit sur les animaux abattus par les chasseurs ou les carcasses étalées dans les marchés ruraux et urbains (Tshikung \& Pongombo, 2009; Makosso et al. 2011; van Vliet et al., 2012; Ngabinzeke et al., 2014; Hette, 2018) ont également indiqué la prédominance des animaux de ces deux ordres. L'on notera aussi que de nombreuses études suggèrent que la prédominance des espèces de rongeurs (lyongo et des conflits humains-faune sous deux aspects: d'une part, les opportunités de contact humains-faune, examinées sur base des données sur la présence de la faune à proximité des lieux d'habitation et des champs, et de l'autre, les dégâts causés par les animaux sauvages dans ces zones. Même si seulement $10 \%$ d'entre eux ont affirmé avoir observé les animaux répertoriés dans les environs (dans un rayon de $3 \mathrm{~km}$ ) des habitations ou des champs, la quasi-totalité des répondants ont évoqué des cas de conflits humainsfaune. En effet, plusieurs enquêtés ont attribué des indices de passage (traces des sabots, restes de nourriture et crottes) et des dégâts aux cultures observés dans les champs de maïs, principale culture de la zone (Useni et al., 2014), à des suidés (Potamochoerus sp.), des antilopes (Tragelaphus spp.) et des primates tels que les grivets de Malbrouck (Chlorocebus cynosuros) et les babouins de Kinda.

II n'existe aucun mécanisme de compensation des dommages causés par les animaux sauvages dans les champs autour du Parc national de Kundelungu. La quasi-totalité des enquêtés ont déclaré recourir à des techniques de contrôle létal telles que le piégeage et l'abattage dans la lutte contre les animaux ravageurs des cultures. A quelques détails près, cette stratégie est en phase avec la loi portant réglementation de la chasse en RDC (Anonyme, 1982) qui dispose (Art. 83) que « toute personne peut se servir de tout moyen de défense contre les animaux sauvages qui, sans avoir été provoqués de quelque manière que ce soit, menacent directement sa vie ou ses biens, la vie ou les biens d'autrui. »

al., 2013) et d'artiodactyles comme le céphalophe bleu, espèce réputée anthropophile (Mathot \& Doucet, 2006; Ngabinzeke et al., 2014), serait indicatrice des milieux perturbés. En effet, en ce qui concerne la zone ciblée par cette étude, l'anthropisation a été confirmée, entre autres, par les impacts humains répertoriés au sein même du Parc national de Kundelungu (Vanleeuwe, 2008). En outre, l'étude de Kabulu et al. (2008) a mis en évidence un processus de fragmentation des forêts claires dans l'ensemble du sud-est de la RDC. De ces évidences, comme dans le cas du Parc national de la Garamba au nord de la RDC (De Merode et al., 2000), ou du « W » au Niger (Moussa \& Amadou, 2014), une approche paysagère d'analyse (Bogaert \& Mahmane, 
2005) des relations spatiales entre le Parc national de Kundelungu et ses milieux environnants pourrait permettre de cerner les facteurs influençant la distribution de la faune au sein de cette aire protégée. Même si une infime proportion des répondants ont déclaré pratiquer la chasse, il convient de souligner ici le rôle des interactions négatives entre la faune et les humains dans l'accentuation de la chasse illicite autour du Parc national de Kundelungu. Les résultats de cette étude suggèrent qu'au vu de leurs perceptions négatives des animaux ravageurs des cultures et des méthodes employées pour les éloigner, notamment le piégeage et la capture, les agriculteurs, représentant la quasi-totalité des enquêtés, sont des chasseurs occasionnels.

\section{CONCLUSION}

Cette étude a cherché à répertorier la faune mammalienne et à rassembler les informations préliminaires sur la chasse et les conflits humainsfaune en périphérie du Parc national de Kundelungu. Les observations directes et indirectes, ainsi que les résultats des enquêtes indiquent que dans les environs des quatre localités enquêtées, parmi les mammifères vus en milieu naturel au cours d'une période allant d'une semaine à trois mois avant ces enquêtes, les répondants ont listé un total de 24 espèces, avec une prépondérance des rongeurs et des artiodactyles. La majeure partie de ces espèces font l'objet d'une chasse de subsistance et sont réputés nuisibles aux cultures.

Les informations préliminaires sur les mammifères répertoriés et les interactions humains-faune illustrent l'importance des périphéries du Parc national de Kundelungu pour la conservation. Cependant, aucun plan de gestion de la faune ou des habitats naturels de ces milieux n'est défini ou mis en œuvre, que ce soit au

\section{REFERENCES BIBLIOGRAPHIQUES}

Anonyme, 1982. Loi $n^{\circ} 82 / 002$ du 28 mai 1982 portant réglementation de la chasse. Journal Officiel, Numéro 11 du 1er Juin 1982.

Anonyme, 2002. Loi nº11/2002 du 29 août 2002 portant Code forestier de la République démocratique du Congo. Journal Officiel de la République Démocratique du Congo, Numéro spécial 6 novembre 2002.

Anonyme, 2004. Arrêté n014/CAB/MIN/ENV/2004 relatif aux mesures d'exécution de la Loi $n^{\circ}$ 82/002 du 28 mai 1982 portant réglementation de la chasse. Journal officiel de la République Démocratique du Congo, Numéro 14.
Au regard des listes rouges de l'UICN, la plupart des espèces répertoriées (Tableau 1) sont évaluées «Préoccupation mineure» à l'échelle de leur aire de distribution. Leur situation locale pourrait cependant être nettement différente. En effet, les dégradations des habitats au sud-est de la RDC (Kabulu et al., 2008; Munyemba \& Bogaert, 2014; Useni et al., 2017) et la forte demande et la consommation des produits de la faune dans les villes proches du Parc comme Lubumbashi (Tshikung \& Pongombo, 2009; Kazaba, 2019) pourraient conduire à des diminutions drastiques des populations animales de cette partie du pays.

niveau de l'aire protégée ou des villages environnants. II sied donc de souligner ici la nécessité des stratégies d'aménagement prenant en compte la configuration et l'utilisation de l'espace géographique partagé entre la faune et les populations locales en périphérie des sites de conservation.

En définitive, la principale leçon tirée de cette étude et, éventuellement, à appliquer à bien d'autres aires protégées en Afrique est que le succès de la conservation nécessitera non seulement l'implication des populations locales et des actions concertées dans la gestion des conflits humains-faune, mais également la caractérisation des activités agricoles et des stratégies locales de lutte contre les animaux réputés nuisibles dans les environs des aires protégées. Ceci permettra d'identifier les chasseurs occasionnels et potentiels et ainsi de mieux évaluer les menaces d'origine anthropique sur des zones d'intérêt écologique mais ne jouissant pas de mesures strictes de protection.

Assani AA, 1999. Analyse de la variabilité temporelle des précipitations (1916-1996) à Lubumbashi (Congo-Kinshasa) en relation avec certains indicateurs de la circulation atmosphérique (oscillation australe) et océanique (El Niño/La Niña). Sécheresse 10(4): 245-252.

Avakoudjo J, Mama A, Toko I, Kindomihou V, Sinsin B, 2014. Dynamique de l'occupation du sol dans le Parc national du W et sa périphérie au nordouest du Bénin. International Journal of Biological and Chemical Sciences 8(6): 26082625. 
Balme G, Slotow R, Hunter LTB, 2010. Edge effects and the impact of non-protected areas in carnivore conservation: Leopards in the Phinda-Mkhuze Complex, South Africa. Animal Conservation 13 (3): 1-9.

Baudron F, Corbeels M, Andersson JA, Sibanda M, Giller KE, 2011. Delineating the drivers of waning wildlife habitat: The predominance of cotton farming on the fringe of protected areas in the Mid-Zambezi Valley, Zimbabwe. Biological Conservation 144: 1481-1493.

Bogaert J et Mahamane A, 2005. Ecologie du paysage: cibler la configuration et l'échelle spatiale. Annales des Sciences Agronomiques du Bénin 7: 39-68.

De Merode E, Hillman-Smith K, Nicholas A, Ndey A, Likango M, 2000. The spatial correlates of wildlife distribution around Garamba National Park, Democratic Republic of Congo. International Journal of Remote Sensing 21(13-14): 2665-2683.

Dupain J, Guislain P, Nguenang GM, De Vleeschouwer K, Van Elsacker L, 2004. High chimpanzee and gorilla densities in a non-protected area on the northern periphery of the Dja Faunal Reserve, Cameroon. Ory 38(02): 209-216.

Geldmann J, Barnes M, Coad L, Craigie ID, Hockings M, Burgess ND, 2013. Effectiveness of terrestrial protected areas in reducing habitat loss and population declines. Biological Conservation 161: 230-238.

Hette S, 2018. Quantification de la viande de brousse prélevée et consommée dans trois villages du sud-est du Cameroun. Travail de Fin d'Etudes (Mémoire de Master). Gemblox Agro-Bio Tech, Université de Liège, Belgique, $73 \mathrm{pp}$.

Inogwabini BI, Omari I, Mbayma AG, Zasy NG, 2005. Protected areas of the Democratic Republic of Congo: A habitat gap analysis to guide the extension of the network. Endangered Species Update 22(2): 71-82.

lyongo LWM, De Cannière C, Ulyel J, Dudu BA, Bukasa K, Verheyen E, Bogaert J, 2013. Effets de lisière et sex-ratio de rongeurs forestiers dans un écosystème fragmenté en République Démocratique du Congo (Réserve de Masako, Kisangani). Tropicultura 31(1) : 3-9.

Jiagho ER, Zapfack L, Banoho LP, Tsayem-Demaze M, Corbonnois J, Tchawa P, 2016. Diversité de la flore ligneuse à la périphérie du Parc national de Waza (Cameroun). VertigO 16(1).

Kabulu DJ, Bamba I, Munyemba KF, Defourny P, Vancutsem C, Nyembwe NS, Ngongo LM, Bogaert J, 2008. Analyse de la structure spatiale des forêts au Katanga. Annales de la Faculté des Sciences Agronomiques 1(2): 1218.

Kazaba KP, 2019. "Non-protected" primates as bushmeat, pets and pests in the southeastern Democratic Republic of Congo. Journal of Threatened Taxa 11(3):13251-13260.

Makosso GV, Massamba J, Massamba A, Silou T, 2011. Consommation de la viande de brousse dans la zone du Parc national de ConkouatiDouli, Congo (Brazzaville): nature du gibier et modalités de consommation. Tropicultura 29(3): 131-137.

Malaisse, F. 2010. How to Live and Survive in Zambezian Open Forest (Miombo Ecoregion). Presses Agronomiques de Gembloux, Gembloux, Belgique, $422 \mathrm{pp}$.

Mathot $L$ et Doucet JL, 2006. Méthode d'inventaire faunique pour le zonage des concessions en forêt tropicales. Bois et Forêts des Tropiques 287(1): 59-70.

Mora C et Sale PF, 2011. Ongoing global biodiversity loss and the need to move beyond protected areas: a review of the technical and practical shortcomings of protected areas on land and sea. Marine Ecology Progress Series 434: 251-266.

Moussa M et Amadou B, 2014. Indicateurs de mesure de la pression anthropique sur les ressources naturelles: exemple de la périphérie du Parc « $W$ » dans la commune rurale de Tamou au Niger. VertigO 14 (1).

Munyemba KF et Bogaert J, 2014. Anthropisation et dynamique spatiotemporelle de l'occupation du sol dans la région de Lubumbashi entre 1956 et 2009. E-Revue UNILU 1: 3-23.

Ngabinzeke JS, Masamba JB, M'Vubu RN, Vermeulen C, 2014. Consommation de produits d'origine animale dans la concession forestière 039/11 de la SODEFOR à Oshwe. Tropicultura 32(3): 147-55.

Saad L, Parmentier I, Colinet G, Malaisse F, Faucon MP, Meerts P, Mahy G, 2012. Investigating the vegetation-soil relationships on the coppercobalt rock outcrops of Katanga (D.R. Congo), 
an essential step in a biodiversity conservation plan. Restoration Ecology 20: 405-415.

Soulama S, Kadeba A, Nacoulma BMI, Traoré S, Bachmann Y, Thiombiano A, 2015. Impact des activités anthropiques sur la dynamique de la végétation de la réserve partielle de faune de Pama et de ses périphéries (sud-est du Burkina Faso) dans un contexte de variabilité climatique. Journal of Applied Biosciences 87: 8047-8064.

Tshikung KM et Pongombo SEW, 2009. Identification et détermination de statut des populations fauniques commercialisées et consommées dans la ville de Lubumbashi, R.D. Congo. Rapport préliminaire. Annales de la Faculté de Médecine Vétérinaire 20: 54-59.

UICN, 2010. Parcs et réserves de la République Démocratique du Congo : évaluation de l'efficacité de gestion des aires protégées. Union internationale pour la Conservation de la Nature/PACO, Ouagadougou, Burkina-Faso, $136 \mathrm{pp}$.

UICN, 2018. The IUCN Red List of Threatened Species. Version 2018-2. http://www.iucnredlist.org. Consultée le 28 janvier 2019.

Useni SY, Kanyenga LA, Assani BL, Ekondo OA, Baboy LL, Ntumba KB, Mpundu MM, Nyembo
$\mathrm{KL}, 2014$. Influence de la date de semis et de la fertilisation inorganique sur le rendement de nouveaux hybrides de maïs (Zea mays L.) à Lubumbashi. Journal of Applied Biosciences 76: 6316- 6325.

Useni SY, Cabala KS, Nkuku KC, Amisi MY, Malaisse F, Bogaert J, Munyemba KF, 2017. Vingt-cinq ans de monitoring de la dynamique spatiale des espaces verts en réponse à l'urbanisation dans les communes de la ville de Lubumbashi (Haut-Katanga, RD Congo). Tropicultura 35(4): 300-311.

van Vliet N, Nebesse C, Gambalemoke S, Akaibe D, Nasi R, 2012. The bushmeat market in Kisangani, Democratic Republic of Congo: implications for conservation and food security. Oryx 46(2): 196-203.

Vanleeuwe $H, 2008$. Large mammals and human impact surveys. Upemba and Kundelungu national parks, Democratic Republic of Congo. WCS/US Fish and Wildlife Service, $30 \mathrm{pp}$.

Western D, Russell S, Cuthill I, 2009. The status of wildlife in protected areas compared to nonProtected areas of Kenya. PLoS One 4(7).

White $F, 1983$. La végétation de l'Afrique : Mémoire accompagné de la carte de la végétation de I'Afrique. ORSTOM-UNESCO: Paris, $384 \mathrm{p}$. 